\title{
Erratum to: Intraoperative detection of viable bone with fluorescence imaging using Visually Enhanced Lesion Scope in patients with bisphosphonate-related osteonecrosis of the jaw: clinical and pathological evaluation
}

\author{
D. Yoshiga ${ }^{1}$ - M. Sasaguri ${ }^{1}$ - K. Matsuo $^{2}$ - S. Kokuryou ${ }^{3}$ - M. Habu ${ }^{1}$ - M. Oda ${ }^{4}$ - M. Kodama ${ }^{1}$.

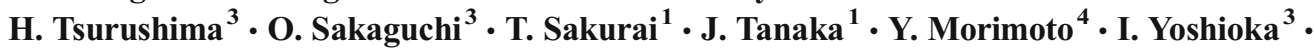 \\ K. Tominaga ${ }^{1}$
}

Published online: 23 June 2015

(C) International Osteoporosis Foundation and National Osteoporosis Foundation 2015

Erratum to: Osteoporos Int

DOI 10.1007/s00198-015-3096-z

The figure and table captions in the original publication were unfortunately incorrect. All tables and figures are reproduced here with the correct legends. The publisher and author regret the inconvenience caused.

The online version of the original article can be found at http://dx.doi.org/ 10.1007/s00198-015-3096-z.

D. Yoshiga

r11yoshiga@fa.kyu-dent.ac.jp

1 Department of Science of Physical Function, Division of

Maxillofacial Surgery, Kyushu Dental University, Fukuoka, Japan

2 Department of Health Promotion, Division of Oral Pathology,

Kyushu Dental University, Fukuoka, Japan

3 Department of Science of Physical Function, Division of Oral

Medicine, Kyushu Dental University, Fukuoka, Japan

4 Department of Science of Physical Function, Division of Oral and

Maxillofacial Radiology, Kyushu Dental University, Fukuoka, Japan 
Table 2 The schematic drawing of the BRONJ treatment protocol in our hospital

Patients with BRONJ visited to our hospital.

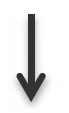

Evaluated areas of osteomyelitis by CT or MRI.

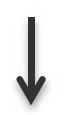

Performed conservative treatment according to AAOMS Position Paper.

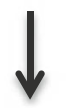

Cases that responses to more than 3-month conservative treatment were bad.

Cases that immediate resumption of Zoledronate administration for cancer treatment were required.

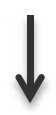

Before surgical treatment, each patient took Minocycline $(200 \mathrm{mg} /$ day) for more than 10 days to control inflammation.

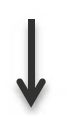

Performed fluorescence-guided resection of necrotic bone including bone fluorescence area.

Complete wound closure with soft tissues are recommended. 
Fig. 1 Case 1: a intraoral photograph shows the exposure of the buccal cortical bone. Panoramic radiogram (b) and computed tomography $(\mathbf{c}, \mathbf{d})$ show sequestrum formation and maxillary sinusitis. Case 2: e panoramic radiogram of the initial visit. f After conservative treatment, bone destruction was enhanced. $\mathbf{g}, \mathbf{h}$ Computed tomography shows sequestrum formation and bone destruction to reach the mandibular canal

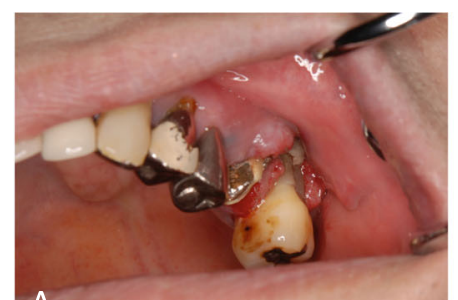

\section{A}
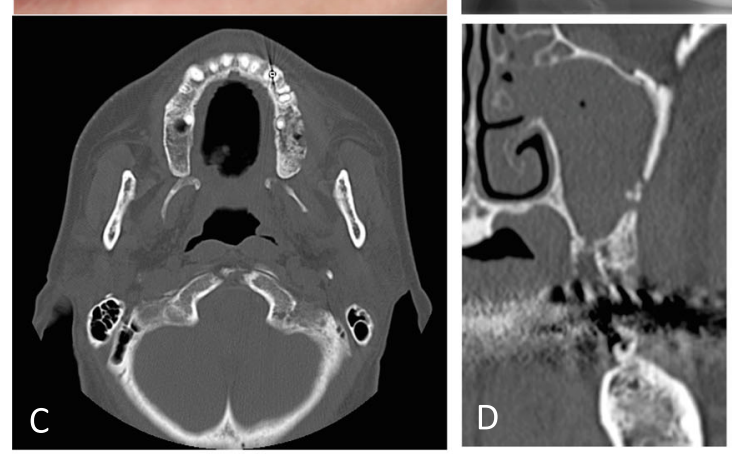

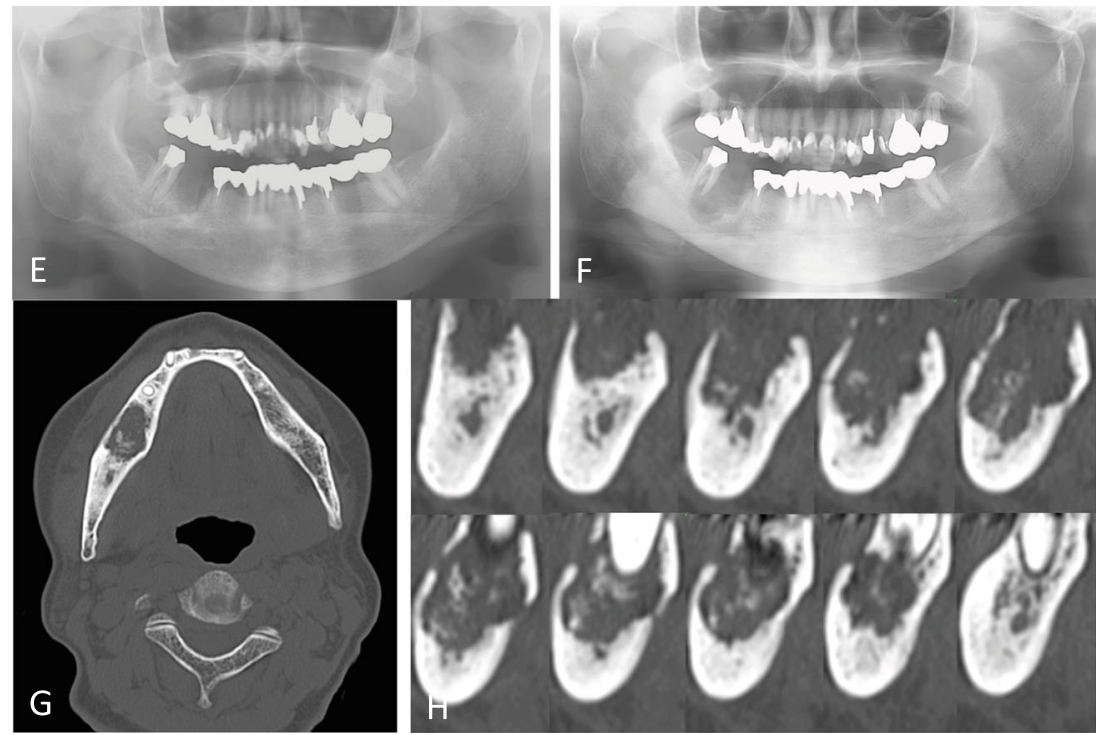


Fig. 2 a Intraoperative picture after bone resection. b Covered with buccal fat pad. c, $\mathbf{d}$ The extent of the unhealthy bone became obvious by use of VELscope $^{\mathbb{R}}$. Arrow heads show bone fluorescence areas

Fig. 3 Clinical picture (a, c) and fluorescence picture $(\mathbf{b}, \mathbf{d}, \mathbf{e})$ of intraoperative fluorescenceguided bone resection by use of VELscope ${ }^{\circledR}$
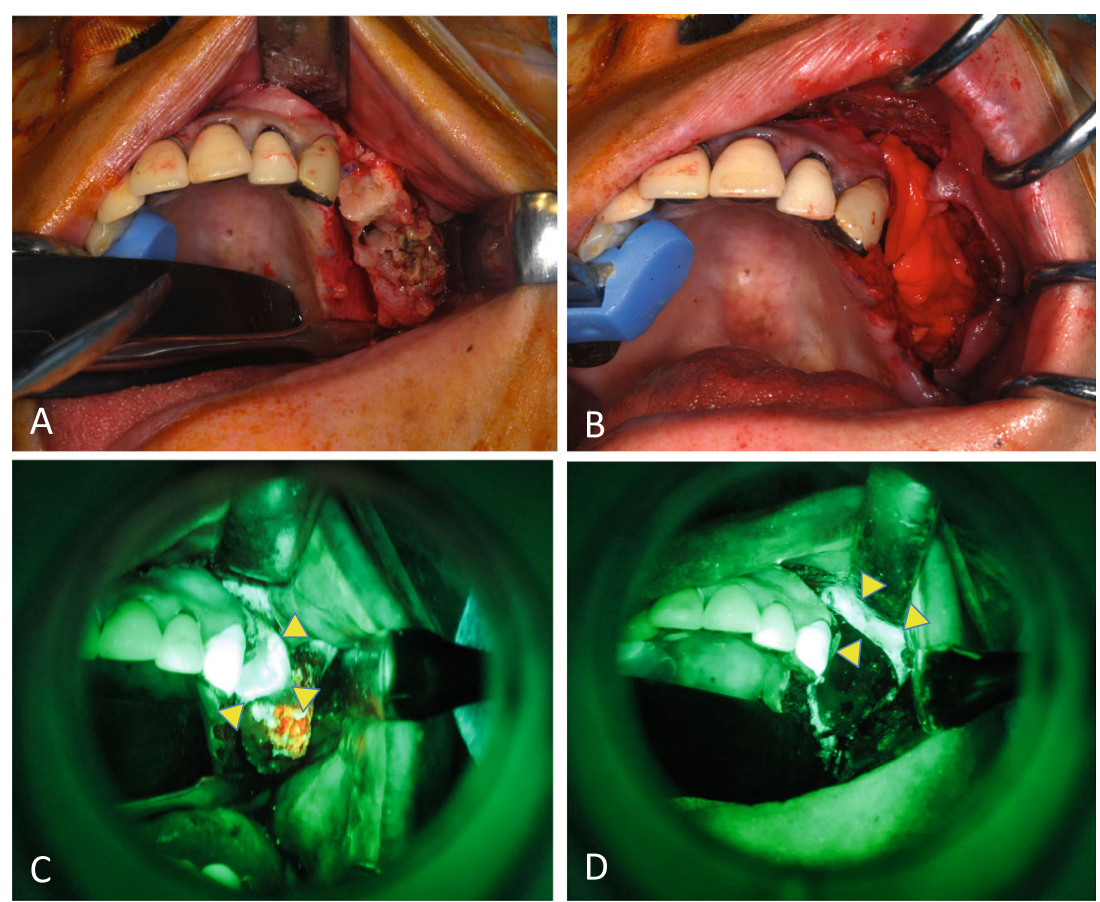
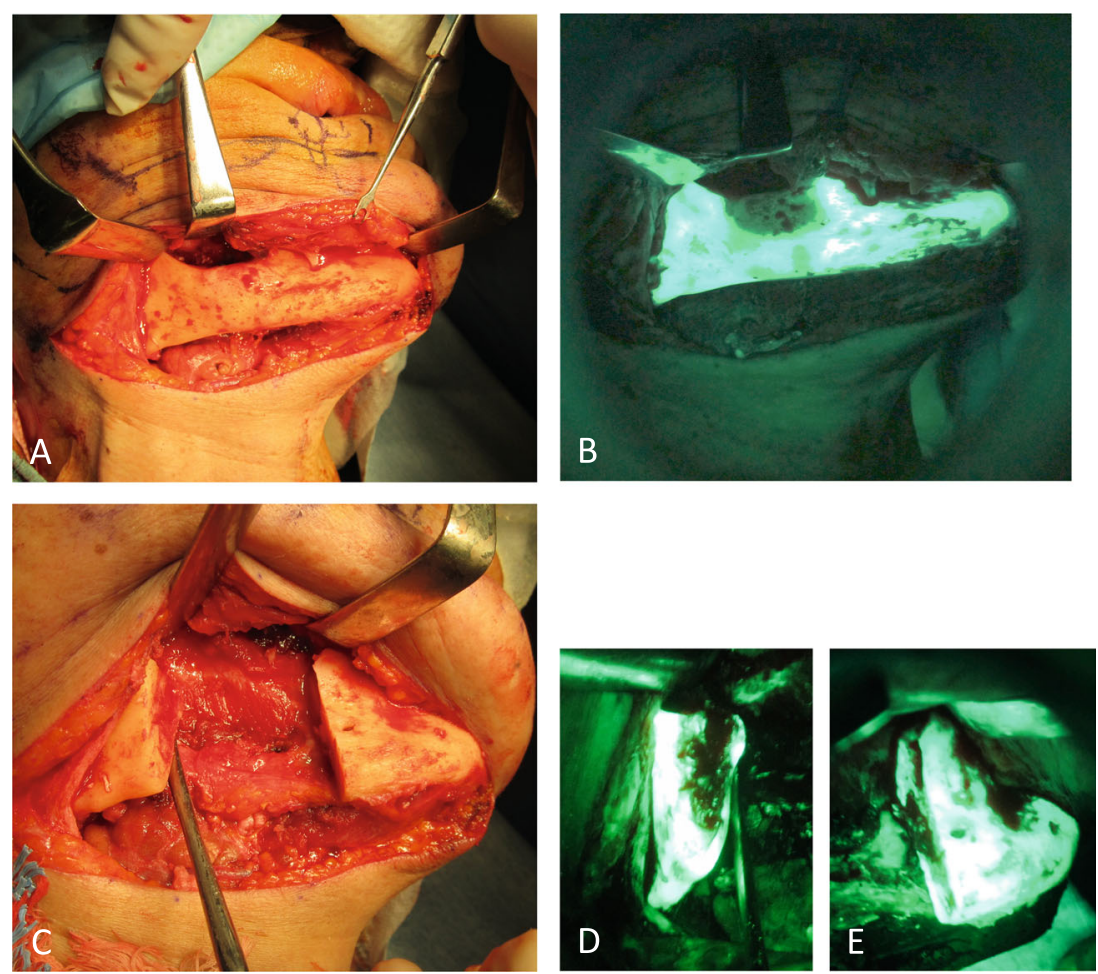
Fig. 4 c, d Histopathological findings of the following section in $\mathbf{a}$ and $\mathbf{b}(\times 40$ magnification)
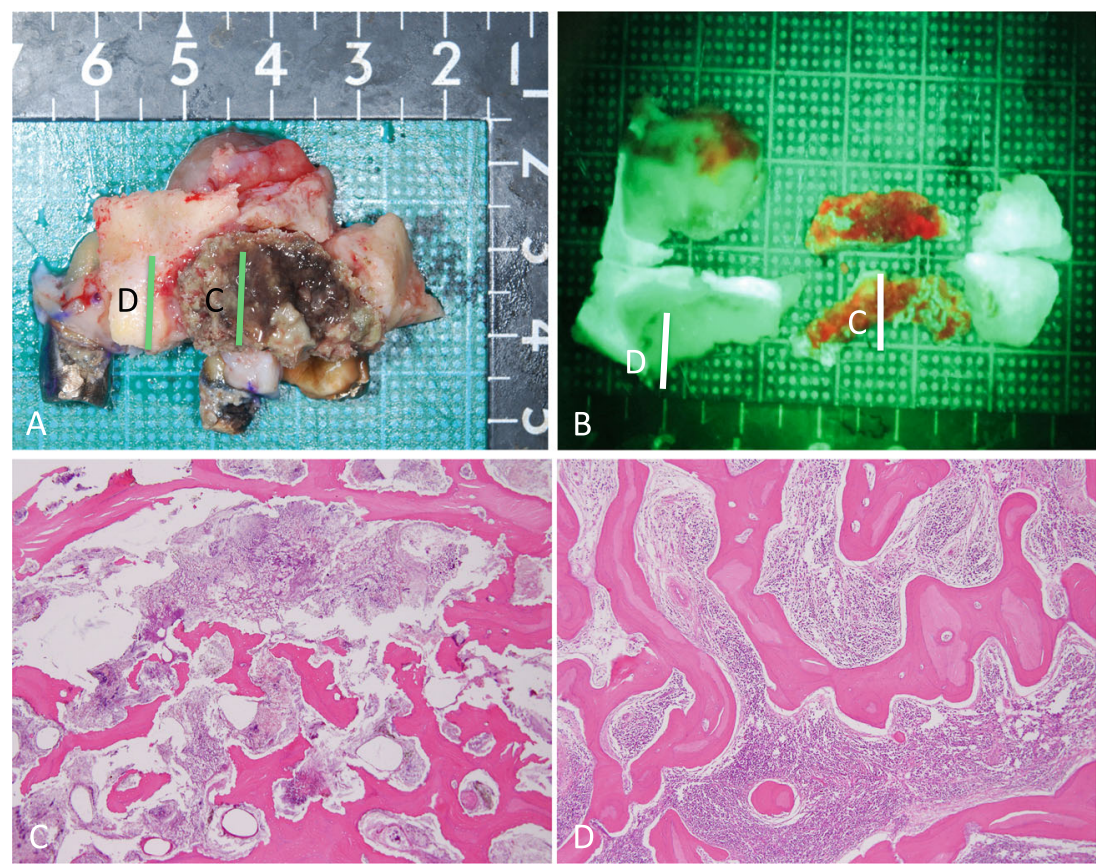

Fig. 5 Clinical pictures and fluorescence pictures of the postoperative resected mandible. a, b Buccal side. c, $\mathbf{d}$ Lingual side. e, f Sagittal cutting surface. g Villanueva bone stain of the sagittal split surface of the postoperative resected mandible (blue minocycline area, red nonminocycline area)
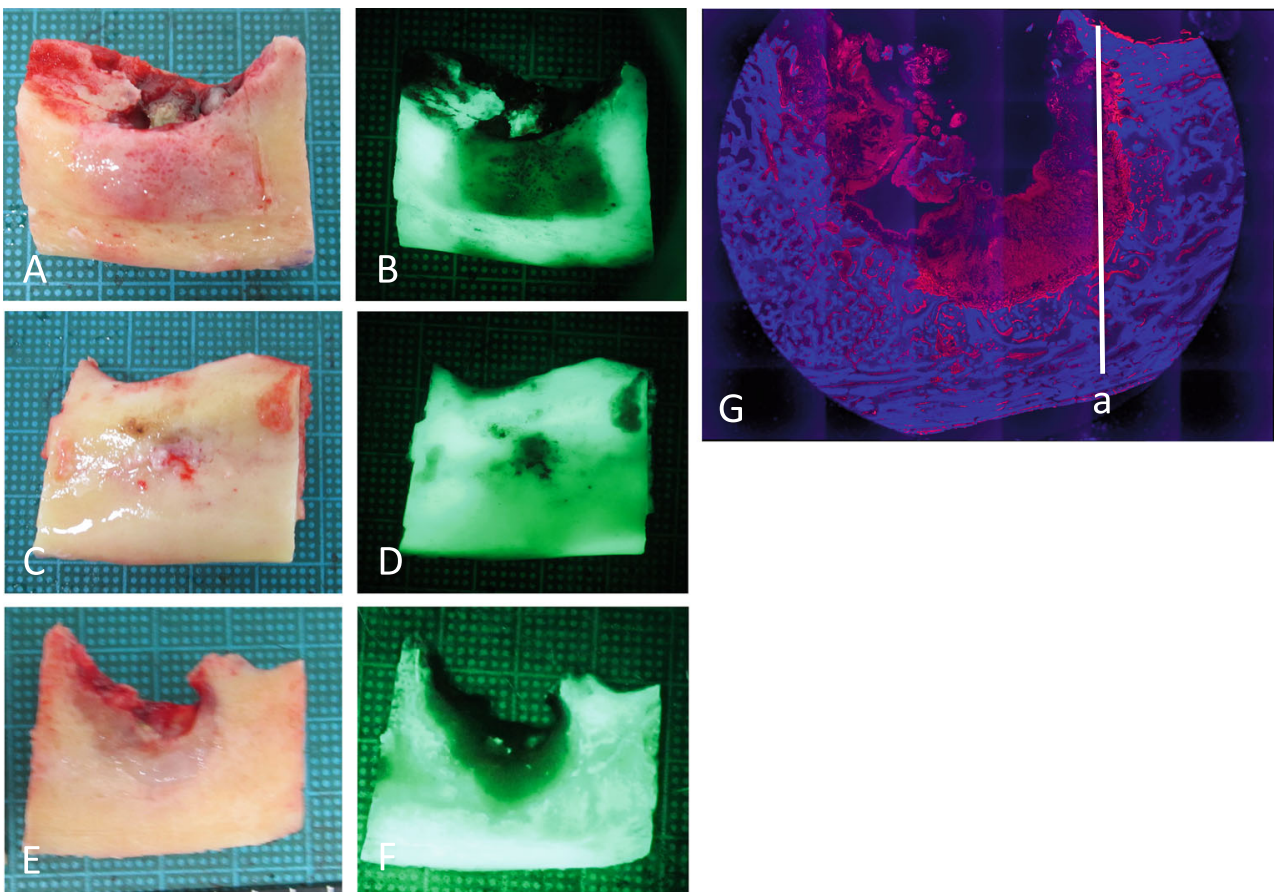
Fig. 6 Histopathological findings of the following section in Fig 5g. The figure shows little compact bone tissue in the substantial portion of cortical bone per low-power field (a) and dense, irregular bony trabeculae bordered by an active layer of osteoblasts and intermingled fibrous tissue with mild

lymphocytic infiltration per highpower field (b) $(\times 40$

magnification)
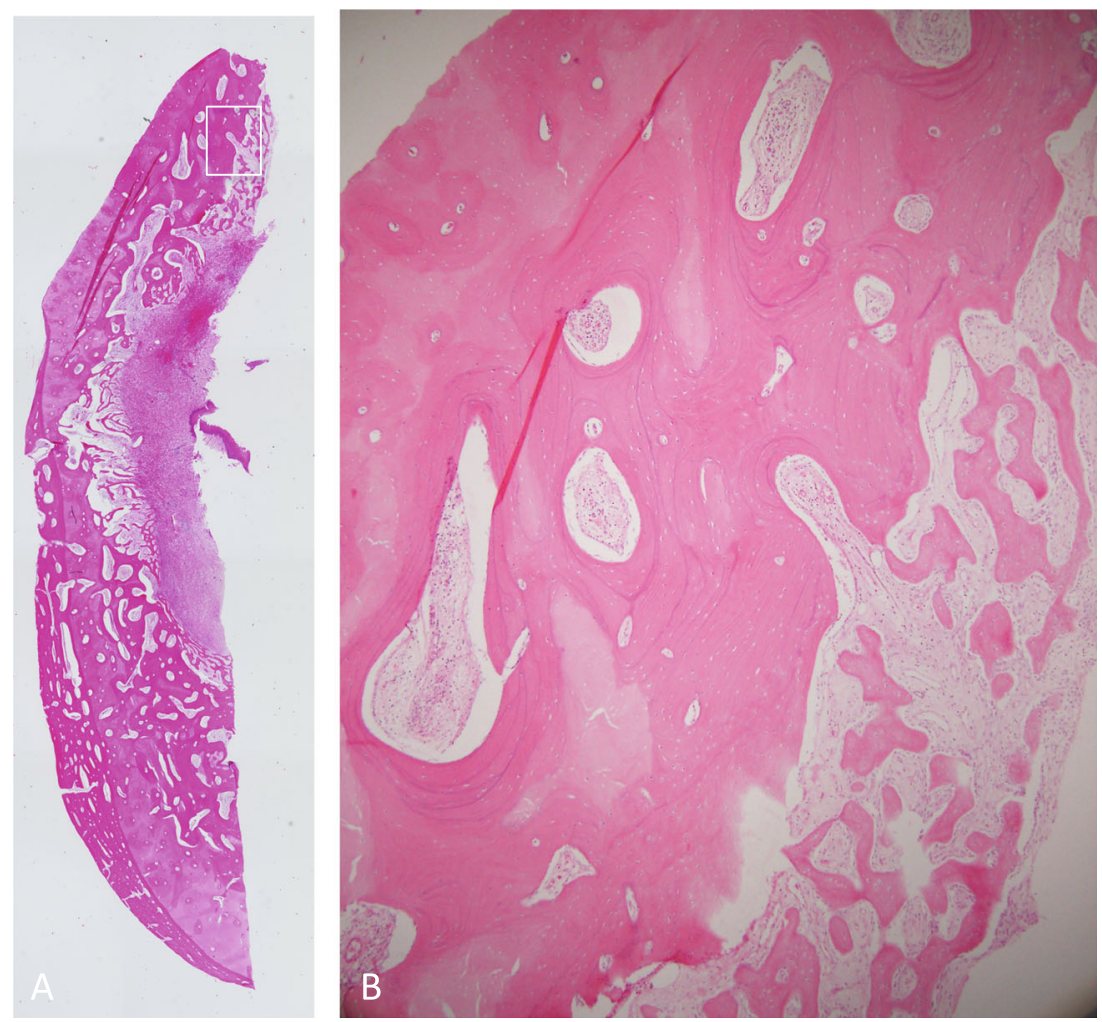